\title{
Community Newsletter: ASH1L mouse model, camouflaging commentary, autism- specific suicidal behaviors survey
}

\section{BY CHELSEY B. COOMBS}

27 JUNE 2021

Hello, and welcome to this week's Community Newsletter! I'm your host, Chelsey B. Coombs, Spectrum's engagement editor.

As a housekeeping note, if you want to continue receiving this newsletter after today, please click here to receive an email from Mailchimp and update your preferences.

We're starting off this week with a tweet from George Mias, associate professor of biochemistry and molecular biology at Michigan State University in East Lansing, who co-wrote a new paper on how the gene ASH1L is tied to both autism and intellectual disability.

New paper! Loss of histone methyltransferase ASH1L in developing mouse \#brain causes autistic-like behaviors. Great work by Jin He Lab (@MSU_BMB) in establishing ASD/ID mouse model

•https://t.co/q8yG7dNDJC via@CommsBio@RobisonLabMSU @gmiaslab @msuresearch\#autism \#neuroscience pic.twitter.com/w8CsIMvDzi

—George Mias (@georgemias) June 18, 2021

The researchers created an ASH1L knockout mouse model, which shows delays in brain development before and after birth. The mice also have behaviors that resemble autism traits seen in people, such as repetitive behaviors and diminished social interaction, and signs of intellectual 


\section{Spectrum | Autism Research News}

https://www.spectrumnews.org

disability, including impaired memory.

The new model, the team writes, will help researchers develop and test "new therapeutic approaches based on the function of ASH1 $\mathrm{L}$ in regulating brain developmental gene expression."

Next up is a tweet from Zack Williams, a graduate student at Vanderbilt University in Nashville, Tennessee, on his new commentary about camouflaging.

Check out my new commentary in JCPP on the construct validity of \#Camouflaging and how researchers can go about validating measures of this important construct. Would love to hear people's thoughts. https://t.co/0wTJnxT13w

—Zack Williams (@QuantPsychiatry) June 18, 2021

Camouflaging in autism has become a hot research topic, but the ways in which researchers currently define and measure it are imprecise, Williams argues.

Researchers often use the self-reported Camouflaging Autistic Traits Questionnaire (CAT-Q), but it's unclear whether CAT-Q subscores or total scores should be used, Williams says, or if the measure is appropriate for all subgroups of autistic people. Future research should determine how distinct camouflaging is from social anxiety and other psychological conditions, he says, and the field should turn to observable behaviors in experimental conditions instead of using self-report questionnaires.

Henry Wood, a research fellow at the University of Southampton in the United Kingdom, tweeted that the article provided "lots of food for thought."

Important article on the construct validity of camouflaging measures. Lots of food for thought ???? https://t.co/xQVMgzJ6QS

— Henry Wood-Downie (@DrHenryWood) June 19, 2021

Meng-Chuan Lai, assistant professor of psychiatry at the University of Toronto in Canada, wrote that the commentary was a "pleasant and enlightening read." 


\section{Spectrum | Autism Research News}

https://www.spectrumnews.org

Insightful and super-constructive suggestions from @QuantPsychiatry about next step research directions to better understand so-called 'camouflaging' in autistic and non-autistic people. Such a pleasant and enlightening read! https://t.co/sle66Y6TtQ

— Meng-Chuan Lai (@mengchuanlai) June 19, 2021

Our last tweet comes from Sarah Cassidy, assistant professor of psychology at the University of Nottingham in the U.K. Her team has created a new version of a suicidal behaviors questionnaire specifically for autistic people, called the Suicidal Behaviors Questionnaire - Autism Spectrum Conditions, or SBQ-ASC.

Delighted to announce publication of of our new tool developed with and for \#autistic adults, to better identify suicidal thoughts and behaviours in research studies:

https://t.co/50LejAm8Xv (free). Summary of this work also in the thread below ????

— Sarah Cassidy (@Sarah_NottsUni) June 21, 2021

Drawing on feedback from autistic people, Cassidy and her colleagues simplified and clarified questions from the standard Suicidal Behaviors Questionnaire, or SBQ-R, adding visual aids to make abstract responses such as "rarely" or "very likely" more concrete. They also added questions to assess the frequency and duration of suicidal thoughts in autistic people.

The new questionnaire is designed for researchers, the team says, and not for clinicians trying to determine a person's risk of future suicide attempts or self-harm.

Rachel Morgan-Trimmer, a neurodiversity consultant and co-founder of the Neurodiversity Association, tweeted that the paper highlights how "academics and others are taking steps to address gaps in services."

This is really good. Not just because the tool itself will identify and prevent harm in autistics, but because academics and others are taking steps to address gaps in services. 


\section{Spectrum | Autism Research News}

https://www.spectrumnews.org

Massive thank you to @Sarah_NottsUni and her team. https://t.co/th3yvKkyaG

— Rachel Morgan-Trimmer (@SparkleClass) June 22, 2021

If you or someone you know is having suicidal thoughts, help is available. Here is a worldwide directory of resources and hotlines that you can call for support.

Finally, we just updated our Twitter lists of Spectrum staffers, freelancers and interns and autism researchers. Check them out if you want to find out what the autism research news community is talking about - and let us know if you'd like to be added.

That's it for this week's Community Newsletter from Spectrum! If you have any suggestions for interesting social posts you saw in the autism research sphere, feel free to send an email to me at chelsey@spectrumnews.org. See you next week!

Cite this article: https://doi.org/10.53053/VDLU2087 